Check for updates

Cite this: RSC Adv., 2017, 7, 37168

\title{
Superhydrophobic surfaces with fluorinated cellulose nanofiber assemblies for oil-water separation $\uparrow$
}

\begin{abstract}
M. Mahbubul Bashar, Huie Zhu, Shunsuke Yamamoto and Masaya Mitsuishi (iD *
A Cellulose nanofiber (CNF) is an amazing nanomaterial produced from ubiquitous sources with outstanding mechanical, chemical, and barrier properties. This report describes facile functionalization of CNF with trichloro $(1 H, 1 H, 2 H, 2 H$-heptadecafluorodecyl)silane (THFS). The paste form CNF was solvent exchanged from initial water to AK-225 (a mixture of 3,3-dichloro-1,1,1,2,2-pentafluoropropane and 1,3-dichloro-1,1,2,2,3-pentafluoropuropane) before surface modification with THFS. The modified CNFs were dispersed uniformly in AK-225. The CNF film, which was prepared using simple drop casting, showed a superhydrophobic surface with a water contact angle of $160^{\circ}$ and oleophilicity with a hexane contact angle of less than $35^{\circ}$. The modified CNF assembly is thermally stable, optically transparent and resistant to corrosive environment (acidic, basic and seawater solutions). The separation of oil and water mixtures was demonstrated using steel mesh coated with modified CNF. Separation efficiency greater than $99 \%$ was achieved by simple gravitational force for hydrocarbons and organic solvents. The asprepared mesh can be used repeatedly more than 50 times with the same efficiency as the initial state.
\end{abstract}

\author{
Received 6th June 2017 \\ Accepted 20th July 2017 \\ DOI: 10.1039/c7ra06316d \\ rsc.li/rsc-advances
}

\section{Introduction}

Research conducted to elucidate superhydrophobic surfaces has drawn much attention from academia to industry for their fascinating wetting behaviour. Research efforts have expanded into their potential applications for self-cleaning, ${ }^{1-4}$ microfluidics, ${ }^{5,6}$ energy conversion, ${ }^{7,8}$ and separation techniques. ${ }^{9-11}$ Superhydrophobic surfaces show water contact angles as high as $150^{\circ}$ along with low sliding angle $\left(<10^{\circ}\right)$. The high contact angle between the liquid drops and surfaces consisting of solid-liquidair interfaces arises from the surface chemistry and engineering control of surface roughness. The fundamental concept of creating superhydrophobic surfaces is imparting low surface energy and design with appropriate roughness. ${ }^{12}$ However, low surface energy is obtainable either by choosing hydrophobic materials or by chemical modification with compounds such as alkanethiols, alkyl or fluorinated organic silanes, and long-chain fatty acids. ${ }^{13}$ Widely diverse approaches have been undertaken for roughening surfaces in earlier studies, including chemical etching, ${ }^{9}$ plasma treatment, ${ }^{14}$ electrospinning, ${ }^{15}$ lithography, ${ }^{16}$ self-assembly, ${ }^{17}$ and hydrothermal ${ }^{18}$ techniques. The duly designed superhydrophobic surfaces exhibit selective absorption of water and organic solvents. Such surfaces can form an organic

Institute of Multidisciplinary Research for Advanced Materials, Tohoku University, 2-1-1 Katahira, Aoba-ku, Sendai 980-8577, Japan.E-mail: masaya@tagen.tohoku.ac. jp

$\dagger$ Electronic supplementary information (ESI) available. See DOI: 10.1039/c7ra06316d solvent sheet from which water rolls off. ${ }^{10}$ As a result, they are able to separate oil and water easily and efficiently from oilwater mixtures. Selective absorption or direct separation of oil from water is one of the important issues in environment protection because of frequent oil spill accidents and the increase of oily wastewater from industrial production. ${ }^{19-25}$ Although there has been noteworthy success in producing superhydrophobic surfaces for selective absorption of liquids, their development remains challenging in terms of creating durable, resistant, and mass-producible bio-based materials. ${ }^{\mathbf{9} 26}$

Cellulose, the most abundant and ubiquitous bio-material on earth, has been used as an engineering material by humans since prehistoric times. It has a hierarchical micronanostructure that includes elementary fibrils and microfibrils. Approximately 36 cellulose chains combine each other through hydrogen bonds to form elementary fibrils. ${ }^{27}$ The elementary fibrils further aggregate into larger microfibrils with typical diameter of 5-50 nm. ${ }^{27-29}$ In terms of molecular design, cellulose is a linear polymer consisting of long chains of an anhydroD-glucopyranose (AGU) unit (Fig. 1(b)). Each AGU repeating unit contains three hydroxyl groups of different moieties. ${ }^{30}$ Therefore, it provides tremendous scope for chemical modifications and reconfigurations. The application of cellulose for fabricating superhydrophobic surfaces is unique because it has an inherent facilitating hierarchical structure.

Numerous reports of the relevant literature have described superhydrophobic surfaces with cellulose either from papers, ${ }^{31}$ filter papers, ${ }^{32}$ or textiles, ${ }^{33,34}$ which have been modified using 


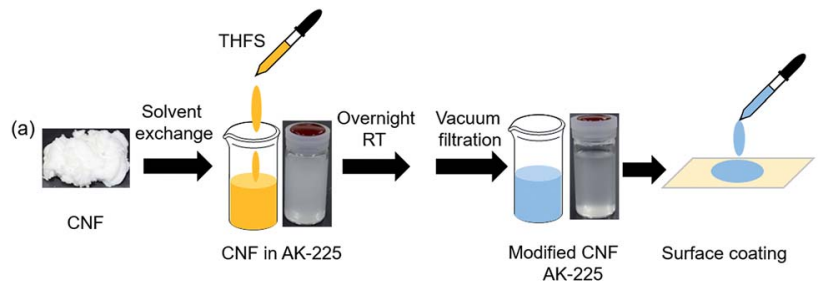

(b)

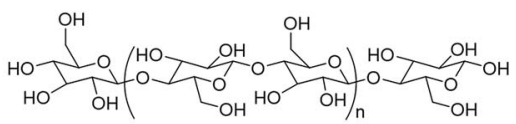

(c) $\quad \mathrm{CF}_{3}\left(\mathrm{CF}_{2}\right)_{7} \mathrm{CH}_{2} \mathrm{CH}_{2}-\underset{\substack{\mathrm{S} \\ \mathrm{S} \\ \mathrm{Cl}}}{\mathrm{Cl}}$

Fig. 1 (a) Schematic illustration of surface modification and surface coating processes and chemical structures of (b) CNF and (c) THFS.

various techniques such as chemical vapour deposition, ${ }^{26}$ dipping method, ${ }^{30}$ and graft on graft method. ${ }^{35}$ Recently, Mertaniemi et al. converted nanofibrillated cellulose using a spray technique. ${ }^{\mathbf{1 2}}$ They succeeded in generating biomimetic porous superhydrophobic structures with nanofibrillated cellulose, which exhibited self-cleaning properties. Many other reports have described for superhydrophobic surfaces with nanocellulose aerogels and cellulose-based textiles intended for oil-water separation..$^{3,36-38}$ Cellulose nanofiber (CNF), which can be isolated from various cellulose sources, exhibits excellent mechanical, optical, and barrier properties. ${ }^{27}$ But surface modification of CNF itself for superhydrophobic surfaces has not been investigated extensively. Moreover, to our knowledge, no report of the relevant literature describes exploration of the superhydrophobicity of CNF for practical applications such as oil-water separation. Therefore, our approach is to modify CNF using a simple one-pot chemical method for superhydrophobic surfaces, steering separation of oil and water mixtures. For this study, we used a fluorinated silane coupling agent (trichloro $(1 \mathrm{H}, 1 \mathrm{H}, 2 \mathrm{H}, 2 \mathrm{H}$-heptadecafluorodecyl) silane, THFS, Fig. 1(c)) as a low surface energy material. Hierarchical roughness with micro-level porosity was generated spontaneously from a simple drop coating assembly of modified CNF. Thereafter, we explored the surface architecture and wetting properties of modified CNF assemblies on different solid substrates. Moreover, transparency and stability towards thermal and chemical exposure of the surface were investigated. Finally, we specifically examined the selective wettability for water and organic solvents and carefully designed an oil-water separation system using a steel mesh. The system exhibits high separation efficiency and higher recyclability for practical applications.

\section{Experimental}

\section{Materials}

CNF was supplied by Daicel Corp., Japan. A fluorinated silane coupling agent, trichloro $(1 H, 1 H, 2 H, 2 H$-heptadecafluorodecyl) silane (THFS) and hydrochlorofluorocarbon solvent, AK-225, were purchased (Wako Pure Chemical Inds. Ltd., Japan). All other chemicals were analytical grade. Steel mesh sheets with
$150 \mu \mathrm{m}$ pore diameter were obtained $(0.1 \times 100 \times 100 \mathrm{~mm}$; The Nilaco Corp., Japan).

\section{Surface modification of CNF and surface coating}

Prior modification of CNF was performed with $60 \%$ sulfuric acid as described in earlier reports to enhance its dispersion ability in organic solvent. ${ }^{39}$ After the paste-like CNF was solvent-exchanged with acetone, chloroform, and AK-225 to remove unbound water, it was dispersed in AK-225 $(30 \mathrm{~mL})$ by stirring for $24 \mathrm{~h}$. The dispersion was diluted by adding $50 \mathrm{~mL}$ AK-225. Then 1.7, 3.4, or $6.8 \mathrm{mM}$ THFS in AK-225 of $20 \mathrm{~mL}$ solution was added dropwise while stirring slowly. Then the reaction mixture was stirred continually for $8 \mathrm{~h}$ at ambient temperature. The final product was washed with AK-225 in a Buchner funnel and was then collected as powder CNF using vacuum filtration. The modified CNF was dried under vacuum and was stored in a refrigerator. Then $0.1 \mathrm{wt} \%$ modified CNF was dispersed in AK-225 by sonication (Ultrasonic homogenizer, $80 \mathrm{~W}$; AS One Corp., Japan). After the solution was drop-casted onto solid substrates, the substrates were dried under vacuum for more than $8 \mathrm{~h}$.

\section{Characterization}

Fourier transform infrared (FT-IR) absorption spectra were taken at spectral resolution of $4 \mathrm{~cm}^{-1}$ using a FTIR spectrometer (FTIR 4200; Jasco Corp.) under a nitrogen atmosphere. The film was prepared on $\mathrm{CaF}_{2}$ substrates for measurements. X-ray photoelectron spectroscopy (XPS) was conducted using a spectrometer (PHI 5600; PerkinElmer Inc.) with a $\mathrm{Mg} \mathrm{K} \alpha \mathrm{X}$-ray source. The detected area was $800 \mu \mathrm{m}^{2}$. To confirm the Si-bonding environment, the glass substrate was coated with a gold layer $(100 \mathrm{~nm})$ using vapour deposition (VK S-200; Osaka Vacuum, Ltd., Japan). Then modified CNF solution was drop-coated upon the gold layer. Elements C, O, $\mathrm{F}$, and $\mathrm{Si}$ were detected at $45^{\circ}$ and $60^{\circ}$ take-off angles. Then the peak position was calibrated with $\mathrm{C}$ 1s peak at $284.5 \mathrm{eV}$. The surface wettability was characterized using a static contact angle measurement instrument (DM 300; Kyowa Interface Science Co., Ltd.) with a $5 \mu \mathrm{L}$ water droplet. Surface morphology of the modified CNF assembly surface was investigated using a field emission scanning electron microscope (FE-SEM, S-4800; Hitachi Ltd., Japan). Thermogravimetric analysis (TGA) was applied using a TGA measurement instrument (thermos plus, TG 8120; Rigaku Corp., Japan.) at a heating rate of $10{ }^{\circ} \mathrm{C} \mathrm{min}^{-1}$. The $\mathrm{N}_{2}$ flow into the combustion chamber was maintained. The film thickness of the modified CNF assembly was measured using a laser scanning microscope (VK-9700; Keyence Co., Japan.). The film optical transparency was analysed using a UV-vis absorption spectrophotometer (V-670; Jasco Corp.).

\section{Results and discussion}

Hydrophilic CNF was modified by a simple one-pot reaction conducted at room temperature. Fig. 1(a) portrays a schematic of a surface functionalization process of CNF (Fig. 1(b)) with THFS (Fig. 1(c)). We selected AK-225 as a dispersion solvent because the solvent allows fluorinated compounds as well as CNF to disperse uniformly. After stepwise solvent substitution, 
surface-modified CNF was dispersed homogeneously in AK-225. The modification was confirmed from FT-IR and XPS results. The most noteworthy point is that two new peaks were observed at $1235 \mathrm{~cm}^{-1}$ and $1205 \mathrm{~cm}^{-1}$ (Fig. 2) attributable to antisymmetric and symmetric stretching of long $\mathrm{CF}_{2}$ groups of THFS. ${ }^{40}$ These two peaks are completely absent for pristine CNF. Furthermore, the relative intensity of these peaks increase as the THFS concentration increases (Fig. S1†). Conversely, modified CNF was accompanied with four absorption bands at $1034,1058,1108$, and $1160 \mathrm{~cm}^{-1}$, indicating the main chain of $\mathrm{C}-\mathrm{O}-\mathrm{C}$ and $\mathrm{C}-\mathrm{O}$ in cellulose molecules, ${ }^{\mathbf{4 1 , 4 2}}$ but their intensity decreases as the amount of THFS increases. Moreover, the characteristic $-\mathrm{OH}$ peak of the cellulose at $3345 \mathrm{~cm}^{-1}$ shifted to a $3285 \mathrm{~cm}^{-1}$ region (Fig. S2 $\dagger$ ) with lower intensity, indicating that new functional groups were incorporated into the $-\mathrm{OH}$ rich cellulose. Similarly, the peak for $-\mathrm{CH}_{2}$ of cellulose main chains at about $2888 \mathrm{~cm}^{-1}$ showed higher intensity in the case of modified CNF, proving the THFS substitution caused by additional $\mathrm{CH}_{2}$ groups. Results show that the CNF surface was decorated with fluorinated moieties of THFS.

Furthermore, the chemical environment of modified CNF was investigated using XPS (Fig. 3). High-resolution spectra of $\mathrm{C} 1 \mathrm{~s}$ obtained before and after modification were observed to confirm the oxidation state. Fig. 3(a) depicts four distinctive peaks for the $\mathrm{C}$ 1s signal. At higher binding energies of 290-295 eV, two peaks attributed to fluorocarbon groups were observed: ${ }^{43,44}$ a peak at $293.8 \mathrm{eV}$ corresponds to $\mathrm{CF}_{3}$ species; the strong peak found at $291.3 \mathrm{eV}$ represents long $\mathrm{CF}_{2}$ chain in fluorinated THFS. The intensity of these two peaks is noteworthy because the longer $\mathrm{CF}_{2}$ group is specified by higher intensity. In contrast, these two peaks are absent in the pristine CNF C 1s XPS spectrum (Fig. 3(b)). A fairly weak peak is observed at $288.6 \mathrm{eV}$ for modified $\mathrm{CNF}$ (Fig. 3(a)), although for pristine CNF, this peak is the strongest (Fig. 3(b)) because all carbon atoms in pristine CNF have at least one $\mathrm{C}-\mathrm{O}$ bonding. ${ }^{45,46}$ Therefore, results indicate that the $\mathrm{C}-\mathrm{O}$ bonding ratio was declined because of the incorporation of fluorinated THFS components. Another peak at $285 \mathrm{eV}$ is ascribed to $\mathrm{CH}_{2}$ groups, both from THFS and CNF main chains. Furthermore, the most crucial observation is the formation of $\mathrm{Si}-\mathrm{O}$ bond at the fibre surface, which was revealed by observing the Si 2 p spectrum

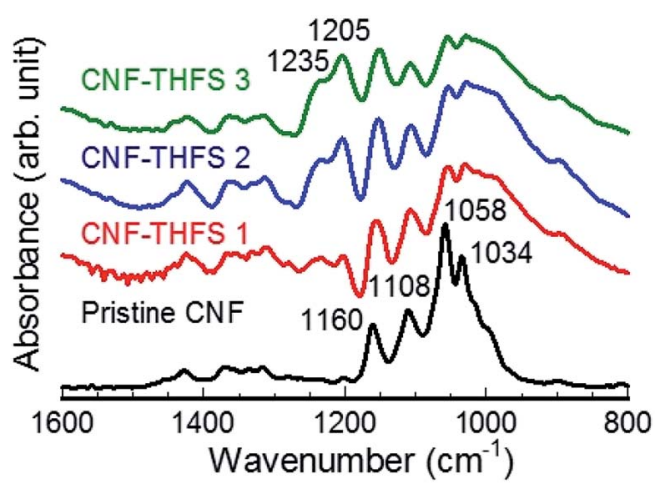

Fig. 2 FT-IR spectra of pristine CNF (black) and modified CNFs with varying amounts of THFS. Numerical values 1,2 , and 3 respectively represent concentrations of THFS: $1.7,3.4$, and $6.8 \mathrm{mM}$.
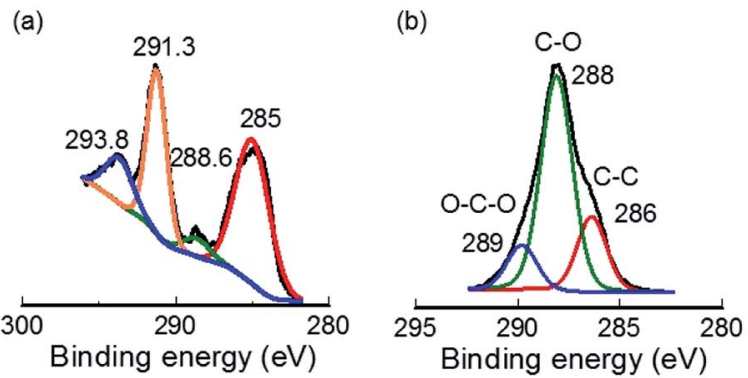

Fig. 3 High-resolution XPS spectra for C 1s (a) modified CNFs and (b) pristine CNFs.

(Fig. S3†). A peak at $103.69 \mathrm{eV}$ indicates a bonding state of Si with $\mathrm{O}$ of hydroxyl group from CNF. ${ }^{47-49}$ Consequently, successful modification of CNF was confirmed.

Fluorinated ligands exhibit very low surface energy. ${ }^{50,51}$ Therefore, we examined the surface free energy (SFE) of the modified CNF assembly surface. The critical surface energy of the modified CNF assembly surface was determined as $17.4 \mathrm{mN} \mathrm{m}^{-1}$ (Fig. S4 $\dagger$ ). Moreover, the component of SFE was evaluated using the Owens and Wendt method. ${ }^{52}$ Also, SFE was calculated for pristine and modified CNF assembly surfaces as well as a self-assembled monolayer (SAM) of THFS (for details see ESI $\dagger$ ). The non-polar and polar components of SFE of the modified CNF assembly surface were evaluated respectively as 10.3 and $3.6 \mathrm{mN} \mathrm{m}^{-1}$, whereas those of the pristine CNF assembly surface were 23.6 and $42.5 \mathrm{mN} \mathrm{m}^{-1}$. In contrast, SAM of THFS revealed 12.6 and $1.0 \mathrm{mN}$ $\mathrm{m}^{-1}$, respectively, for non-polar and polar counterparts. These results prove that the modified CNF assembly surface has very low surface energy, making it ideal for superhydrophobic surfaces. Furthermore, the adhesion force of water droplet on the modified CNF assembly surface was confirmed qualitatively using a static contact angle measurement system..$^{53}$ A $5 \mu \mathrm{L}$ water droplet was placed on the modified CNF assembly surface. No adhesion was observed after loading and unloading processes (Fig. S5†). The water droplet can bounce on the CNF assembly surface (Fig. S6 $\dagger$ ), though its sliding angle was relatively high (on glass substrates): $16.7 \pm 2^{\circ}$. These results suggest good water-repelling properties of the modified CNF assembly.

Another important criterion for superhydrophobic surface is surface roughness originating from surface geometrical architectures. Fig. 4(a) presents a low magnification image showing that aggregation of fibres produced bundles or a sheet-like appearance. This sheet formation results from the solvent exchange process that occurs during a modification step. Because the image is magnified, fibrillar structures with hierarchical alignment are visible (Fig. 4(b)). Moreover, highly porous surfaces were confirmed to have 1-5 $\mu \mathrm{m}$ average pore diameter (Fig. 4(c)). Furthermore, a 'nanostair'like appearance was observed on a few hundred nanometer scale (Fig. 4(d)). This micro-nanostructure of modified CNF assembly surfaces is dependent on the amount of the dropcast suspension, which was controlled by varying the concentration of the modified CNF (Fig. S7†). Therefore, 


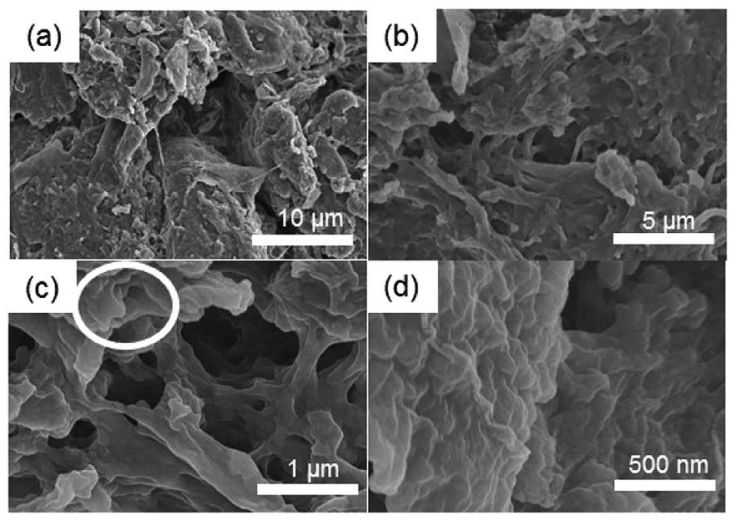

Fig. 4 FE-SEM images of modified CNF on Si substrates with different magnifications: (a) fibre aggregation producing fibre bundles, (b) fibrillar structure with hierarchical alignment, (c) hierarchical porous structure with pore diameters of 1-5 $\mu \mathrm{m}$, and (d) nanostair appearance in modified CNF at high magnification of the circled area in (c).

surface morphology of modified CNFs revealed a hierarchical micro-nanostructure having considerable porosity, which ultimately imparts extremely high roughness to the surface.

Consequently, our modified CNF assembly surface exhibited very low surface energy with appropriate roughness. As a result, it is highly desired that such a surface exhibits unusual wetting behaviour. Fig. 5 displays an example of water contact angles (WCAs) of pristine CNF assembly (Fig. 5(a)) and modified CNF assembly (Fig. 5(b)) on silicon substrates. The WCA value of modified CNF assembly surface is $160 \pm 2^{\circ}$, producing an almost spherical shape with a $5 \mu \mathrm{L}$ water droplet. In contrast, the pristine $\mathrm{CNF}$ assembly surface is hydrophilic, showing a WCA value of $13 \pm 2^{\circ}$. This high water contact angle of the modified CNF assembly surface is explained by the Wenzel and Cassie-Baxter models. The Wenzel model describes wetting phenomena of a liquid for a solid rough surface. ${ }^{32}$ According to this model, the roughness plays an extremely important role in the wetting of solid. Cassie-Baxter equations can be written as

$$
\cos \theta_{\mathrm{r}}=f(\cos \theta+1)-1
$$

(a)

(b)
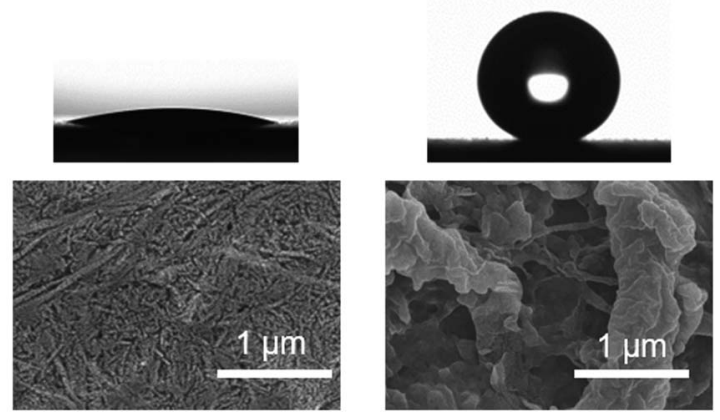

Fig. 5 Photographs of water droplet on substrates coated with (a) pristine CNF and (b) modified CNF with their corresponding SEM images. where $\theta_{\mathrm{r}}$ and $\theta$ respectively denote the contact angles of a rough surface and a flat surface; $f$ represents the fractional areas of a liquid droplet in contact with air on the surface. The CassieBaxter equation is associated with the area fraction covered by the liquid droplet to solid and air. ${ }^{32}$ In the case of the modified $\mathrm{CNF}$ assembly surface, the value of the air-liquid fractional area was calculated as $90.8 \%$, which accounted for the effective surface modification of CNFs with fluorinated THFS (see ESI and Fig. S8†).

A particularly beneficial property of superhydrophobic surfaces is their optical transparency. The integration of optical transparency into superhydrophobic surfaces is challenging because the surface roughness is expected to be less than visible light wavelengths. ${ }^{13}$ Our modified CNF assembly surface roughness is so high because of the aggregation of fibres, which limits the detection of surface roughness using atomic surface microscopy (AFM) technique. Even our modified CNF assembly surface showed a certain limit of transparency. Fig. 6(a) shows visual transparency on a glass substrate coated with modified CNF. The film thickness was confirmed as about 9.3 and 15.0 $\mu \mathrm{m}$ (Fig. S9 $\dagger$ ) for two assemblies with respective transparencies at $500 \mathrm{~nm}$ wavelength of 58.3 and $57.0 \%$ (Fig. 6(b)). These two surfaces respectively showed WCAs of $160^{\circ}$ and $165^{\circ}$. The latter exhibited a high WCA value because of higher surface roughness, which is in agreement with the theory of superhydrophobic surfaces.

We investigated the stability of as-prepared superhydrophobic surfaces for thermal and chemical exposure. To hot water, artificial seawater and strong acidic and basic solutions for 12 immersion cycles with 5 min dipping: the total immersion time was $1 \mathrm{~h}$. Fig. 7(a) depicts WCA values for different cycles dipped in hot water at $60{ }^{\circ} \mathrm{C}$ and $90{ }^{\circ} \mathrm{C}$. In both cases, the average WCA values were found to be higher than $150^{\circ}$, signifying the stability of superhydrophobicity at higher temperatures. The reason for robust superhydrophobicity with hot water on modified CNF assemblies was confirmed using a thermogravimetric analysis of pristine, solvent-exchanged and THFS-modified CNFs (Fig. S10†). The modified CNF exhibited better thermal stability than the solvent-exchanged CNF. In fact, the CNF thermal stability was enhanced from its initial $80{ }^{\circ} \mathrm{C}$ to $220{ }^{\circ} \mathrm{C}$, which is an effective working temperature of cellulosic materials. ${ }^{54,55}$ The improved thermal stability derives from the incorporation of fluorinated ligands into the CNF surface, which might crosslink CNFs. It is (a)

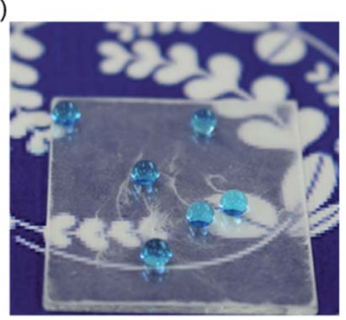

(b)

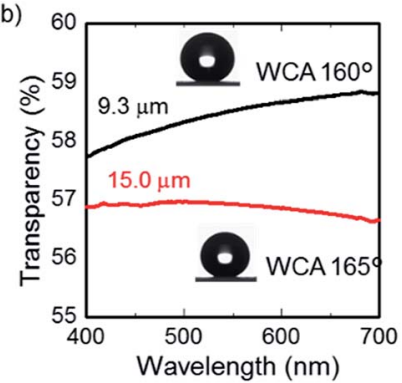

Fig. 6 (a) Photograph and (b) optical transmission spectra of $9.3 \mu \mathrm{m}$ (black) and $15.0 \mu \mathrm{m}$ (red) thick modified CNF assemblies. 
(a)
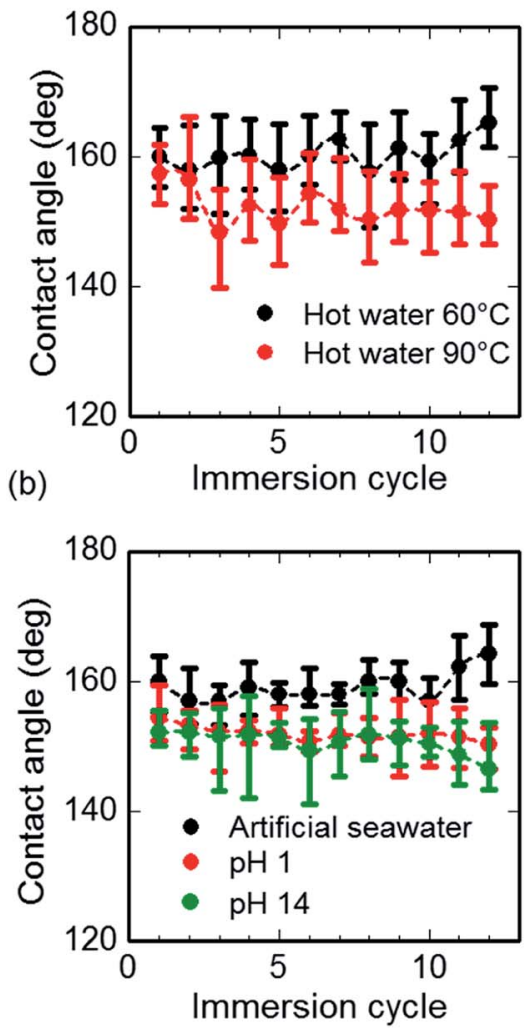

Fig. 7 WCA values on modified CNF assembly surfaces as a function of the number of immersion cycles for (a) hot water at $60^{\circ} \mathrm{C}$ (black) and $90{ }^{\circ} \mathrm{C}$ (red), and (b) artificial seawater (black), acidic (red), and alkaline (green) water solutions.

noteworthy that pristine CNFs exhibited better thermal stability at temperatures higher than $400{ }^{\circ} \mathrm{C}$. The presence of water might affect the hydrogen bonding interactions of CNFs that accounted the higher thermal stability.

The chemical stability of the as-prepared modified CNF assembly was investigated by measuring WCA values for highly alkaline, saline, and acidic solutions. Fig. 7(b) presents WCA values for artificial seawater, highly alkaline (pH 14) and highly acidic ( $\mathrm{pH}$ 1) water solutions. In all cases, the WCA values were recorded as greater than $150^{\circ}$. It is noteworthy that the strongly alkaline solutions exhibited a lower contact angle, indicating less stability towards alkaline conditions than towards the acidic environment. However, superhydrophobicity remained unaffected.

Next we devote attention to the contact angle of modified CNF assemblies for hydrocarbons. Fig. 8 shows the selective wettability towards water and octane for $20 \mu \mathrm{L}$ droplet. Octane droplets, shown as orange, spread on the surface whereas water droplets, blue colour, produced an almost spherical shape. Additionally, the wettability of organic hydrocarbons is presented in Fig. S11, $\dagger$ with a lower contact angle than that of water. The phenomena reflect the superhydrophobicity and oleophilicity of the modified CNF assembly surfaces.

As we reported earlier, our modified CNF assembly surface showed selective wettability with robustness in various severe

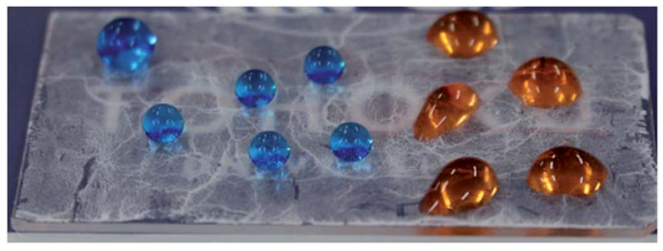

Fig. 8 Selective wettability of modified CNF surface. A $20 \mu \mathrm{L}$ octane (orange color) droplet spreads over the surface while water droplet (blue color) roll off on the surface.

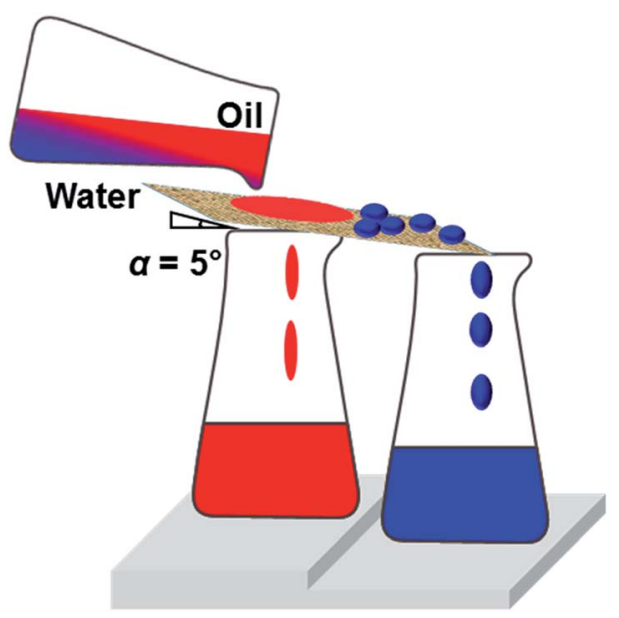

Fig. 9 Schematic illustration of oil-water separation by a metal mesh coated with modified CNF. The mesh pore size was $150 \mu \mathrm{m}$. The water droplet easily bounces and rolls off at a sliding angle $5^{\circ}$ while oil penetrates through the mesh by gravitational force.

environmental conditions. It can therefore be regarded as competent for one solution of oil spillage from marine water. Fig. 9 depicts a schematic illustration of oil-water separation by metal mesh coated with modified CNF assemblies. The mesh pore diameter was $150 \mu \mathrm{m}$. The mesh plane was tilted by $5^{\circ}$, facilitating the rolling off of water droplets from the surface: oil (various hydrocarbons) penetrates through the mesh readily by gravitational force.

SEM images of uncoated and coated meshes are displayed, respectively, in Fig. S12(a) and S12(b). $\dagger$ The uncoated mesh is smooth, showing an average WCA value of $110^{\circ}$. However, the coated mesh is fashioned with modified CNFs in hierarchical micro-nano alignment (inset), facilitating repulsion with a high WCA value of $162^{\circ}$. Fig. 10(a) presents a photograph of the separation process. The water droplet (blue) remains on the coated steel mesh, whereas octane (orange) permeates through the mesh. Additionally, a movie showing separation is presented in ESI. $\dagger$

After the filtration process, almost pure octane and water were collected separately (Fig. 10(b)). In the separated water, however, some oil was present, because the water droplets rolled off after oil wetted the surface beforehand. Nevertheless, the separated oil contains no residual water, which was confirmed by addition of methylene blue dyes. Moreover, separation was 
(a)

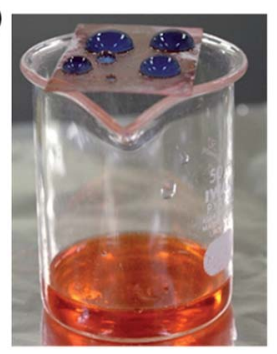

(b)

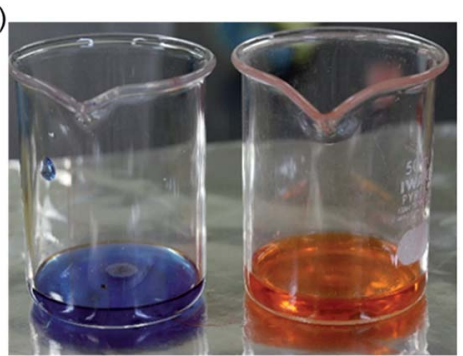

(c)

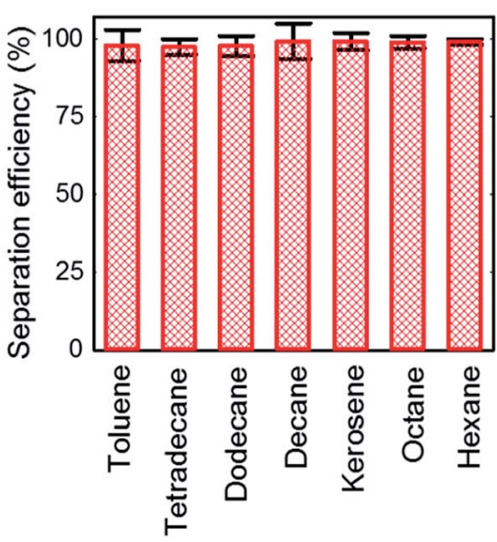

Fig. 10 Photographs of separation process: (a) water droplet (blue) standing on the coated steel mesh whereas octane (orange) penetrated through the mesh stored in the beaker, (b) collected water and octane after separation, and (c) separation efficiency of the coated steel mesh for different organic solvents with greater than $90 \%$ efficiency.

conducted for many hydrocarbons and organic solvents including tetradecane, dodecane, decane, octane, kerosene, hexane, and toluene, keeping the volumetric ratio of water to oil as $1: 1$. A total volume of $20 \mathrm{~mL}$ oil-water mixture was separated. The separation efficiency was estimated based on the volume of water collected after separation according to an earlier report. ${ }^{11}$ Fig. 10 (c) expresses the separation efficiency for each organic solvent. The efficiency was calculated as $99 \%$ for decane, octane, and hexane, and kerosene. It is noteworthy that the designed coated mesh can separate higher hydrocarbons such as tetradecane, dodecane, and the organic solvent toluene with more than 97\% efficiency (Fig. S13†), although they exhibited a contact angle greater than $100^{\circ}$. The modified coated mesh is highly robust in terms of the separation cycle. In fact, it can be used more than 50 times with the same separation efficiency and no significant fouling by organic solvents was observed. Even after 50 cycles the coated mesh is available for the next step with no additional treatment such as washing and cleaning. Further investigation, addressing wear and abrasion resistance $^{56}$ will offer exciting opportunities for practical applications.

\section{Conclusions}

We have demonstrated a simple and convenient approach to modify CNF for the preparation of superhydrophobic surfaces,

potentially enabling efficient oil-water separation. CNF surfaces were modified with fluorinated silane coupling agents in homogeneous dispersion. Modified CNFs took hierarchical assembly structures on solid supports. They are thermally stable and resistant to extreme acidic and alkaline conditions. The steel mesh coated with modified CNFs can separate oil and water efficiently and quickly from mixtures with 99\% separation efficiency simply using gravitational force. The separation process can be repeated more than 50 times with equal performance. Consequently, our modified CNFs are expected to be sustainable and effective materials for the separation of oil and water. They are promising for scalable fabrication with low cost, high separation efficiency and excellent durability as well as for industrial applications for the separation of oil from seawater.

\section{Acknowledgements}

We thank Daicel Corp. for supplying cellulose nanofiber. The work was supported by Grants-in-Aid for Scientific Research (B) (16H04197) and for Young Scientists (16K17953) from the Japan Society for the Promotion of Science (JSPS). The work was also supported by the Research Program of "Dynamic Alliance for Open Innovation Bridging Human, Environment and Materials" in "Network Joint Research Centre for Materials and Devices" (MEXT). Furthermore, the authors are grateful to Ms. Sayaka Ogawa, IMRAM, Tohoku University, for the use of an Xray photoelectron spectroscope. The Central Analytical Facility of IMRAM is also gratefully acknowledged for providing the AFM, SEM, and TGA instruments.

\section{Notes and references}

1 C. Schlaich, L. Cuellar Camacho, L. Yu, K. Achazi, Q. Wei and R. Haag, ACS Appl. Mater. Interfaces, 2016, 8, 29117.

2 Z. Chu, Y. Feng and S. Seeger, Angew. Chem., Int. Ed., 2015, 54, 2328.

3 Z. Xue, Y. Cao, N. Liu, L. Feng and L. Jiang, J. Mater. Chem. A, 2014, 2, 2445.

4 X. Zhang, Z. Li, K. Liu and L. Jiang, Adv. Funct. Mater., 2013, 23, 2881.

5 L. Ionov, N. Houbenov, A. Sidorenko, M. Stamm and S. Minko, Adv. Funct. Mater., 2006, 16, 1153.

6 L. Zhang, Z. Zhang and P. Wang, NPG Asia Mater., 2012, 4, e8. 7 S. Nishimoto and B. Bhushan, RSC Adv., 2013, 3, 671.

8 N. Miljkovic, R. Enright and E. N. Wang, ACS Nano, 2012, 6, 1776.

9 C. Wang, T. Yao, J. Wu, C. Ma, Z. Fan, Z. Wang, Y. Cheng, Q. Lin and B. Yang, ACS Appl. Mater. Interfaces, 2009, 1, 2613.

10 C. R. Crick, J. A. Gibbins and I. P. Parkin, J. Mater. Chem. A, 2013, 1, 5943.

11 X. Zhou, Z. Zhang, X. Xu, F. Guo, X. Zhu, X. Men and B. Ge, ACS Appl. Mater. Interfaces, 2013, 5, 7208.

12 H. Mertaniemi, A. Laukkanen, J. E. Teirfolk, O. Ikkala and R. H. A. Ras, $R S C$ Adv., 2012, 2, 2882.

13 X. Zhang, F. Shi, J. Niu, Y. Jiang and Z. Wang, J. Mater. Chem., 2008, 18, 621. 
14 V. Zorba, E. Stratakis, M. Barberoglou, E. Spanakis, P. Tzanetakis, S. H. Anastasiadis and C. Fotakis, Adv. Mater., 2008, 20, 4049.

15 L. Wang, S. Yang, J. Wang, C. Wang and L. Chen, Mater. Lett., 2011, 65, 869.

16 J. Y. Shiu, C. W. Kuo, P. Chen and C. Y. Mou, Chem. Mater., 2004, 16, 561.

17 J. Wang, Z. Shi, J. Fan, Y. Ge, J. Yin and G. Hu, J. Mater. Chem., 2012, 22, 22459.

18 H. Li, M. Zheng, L. Ma, C. Zhu and S. Lu, Mater. Res. Bull., 2013, 48, 25.

19 B. Wang, W. Liang, Z. Guo and W. Liu, Chem. Soc. Rev., 2015, 44, 336.

20 Z. Xue, S. Wang, L. Lin, L. Chen, M. Liu, L. Feng and L. Jiang, Adv. Mater., 2011, 23, 4270.

21 A. K. Kota, G. Kwon, W. Choi, J. M. Mabry and A. Tuteja, Nat. Commun., 2012, 3, 1025.

22 S. Gao, J. Sun, P. Liu, F. Zhang, W. Zhang, S. Yuan, J. Li and J. Jin, Adv. Mater., 2016, 28, 5307.

23 W. Zhang, Z. Shi, F. Zhang, X. Liu, J. Jin and L. Jiang, Adv. Mater., 2013, 25, 2071.

24 J. Li, L. Yan, Y. Zhao, F. Zha, Q. Wang and Z. Lei, Phys. Chem. Chem. Phys., 2015, 17, 6451.

25 Y. Si, Q. Fu, X. Wang, J. Zhu, J. Yu, G. Sun and B. Ding, ACS Nano, 2015, 9, 3791.

26 S. Li, H. Xie, S. Zhang and X. Wang, Chem. Commun., 2007, 46, 4857.

27 R. J. Moon, A. Martini, J. Nairn, J. Simonsen and J. Youngblood, Chem. Soc. Rev., 2011, 40, 3941.

28 M. R. K. Sofla, R. J. Brown, T. Tsuzuki and T. J. Rainey, Adv. Nat. Sci.: Nanosci. Nanotechnol., 2016, 7, 035004.

29 M. T. Postek, A. Vladár, J. Dagata, N. Farkas, B. Ming, R. Wagner, A. Raman, R. J. Moon, R. Sabo, T. H. Wegner and J. Beecher, Meas. Sci. Technol., 2010, 22, 024005.

30 A. G. Cunha and A. Gandini, Cellulose, 2010, 17, 875.

31 H. Yang and Y. Deng, J. Colloid Interface Sci., 2008, 325, 588.

32 X. Huang, X. Wen, J. Cheng and Z. Yang, Appl. Surf. Sci., 2012, 258, 8739.

33 S. Michielsen and H. J. Lee, Langmuir, 2007, 23, 6004.

34 H. F. Hoefnagels, D. Wu, G. de With and W. Ming, Langmuir, 2007, 23, 13158.

35 D. Nyström, J. Lindqvist, E. Östmark, A. Hult and E. Malmström, Chem. Commun., 2006, 34, 3594.
36 J. T. Korhonen, M. Kettunen, R. H. A. Ras and O. Ikkala, ACS Appl. Mater. Interfaces, 2011, 3, 1813.

37 N. T. Cervin, C. Aulin, P. T. Larsson and L. Wågberg, Cellulose, 2012, 19, 401.

38 C. H. Xue, P. T. Ji, P. Zhang, Y. R. Li and S. T. Jia, Appl. Surf. Sci., 2013, 284, 464.

39 W. Bai, J. Holbery and K. Li, Cellulose, 2009, 16, 455.

40 M. Kobayashi, K. Tashiro and H. Tadokoro, Macromolecules, 1975, 8, 158.

41 W. Chen, H. Yu, Y. Liu, Y. Hai, M. Zhang and P. Chen, Cellulose, 2011, 18, 433.

42 C. Chung, M. Lee and E. K. Choe, Carbohydr. Polym., 2004, $58,417$.

43 T. Kajiyama, S. Ge, K. Kojio and A. Takahara, Supramol. Sci., 1996, 3, 123.

44 T. Ishida, S. Yamamoto, W. Mizutani, M. Motomatsu, H. Tokumoto, H. Hokari, H. Azehara and M. Fujihira, Langmuir, 1997, 13, 3261.

45 Y. Habibi, L. Foulon, V. Aguié-Béghin, M. Molinari and R. Douillard, J. Colloid Interface Sci., 2007, 316, 388.

46 Y. Habibi, I. Hoeger, S. S. Kelley and O. J. Rojas, Langmuir, 2009, 26, 990.

47 A. Ishizaka and Y. Shiraki, J. Electrochem. Soc., 1986, 133, 666.

48 A. Bansal, X. Li, I. Lauermann, N. S. Lewis, S. I. Yi and W. H. Weinberg, J. Am. Chem. Soc., 1996, 118, 7225.

49 J. F. Moulder, Handbook of X-ray photoelectron spectroscopy: a reference book of standard spectra for identification and interpretation of XPS data, ed. J. Chastain and R. C. King, Physical Electronics, Eden Prairie, MN, 1995, pp. 84-85.

50 K. Li, P. Wu and Z. Han, Polymer, 2002, 43, 4079.

51 S. Borkar, K. Jankova, H. W. Siesler and S. Hvilsted, Macromolecules, 2004, 37, 788.

52 D. K. Owens and R. C. Wendt, J. Appl. Polym. Sci., 1969, 13, 1741.

53 Y. Gao, T. Chen, S. Yamamoto, T. Miyashita and M. Mitsuishi, ACS Appl. Mater. Interfaces, 2015, 7, 3468.

54 H. Yang, R. Yan, H. Chen, D. H. Lee and C. Zheng, Fuel, 2007, 86, 1781.

55 L. Chen, J. Y. Zhu, C. Baez, P. Kitin and T. Elder, Green Chem., 2016, 18, 3835.

56 I. S. Bayer, Coating, 2017, 7, 12. 